\title{
Nationalismus und Arbeitsmarktintegration in der BRD (alt)
}

\author{
David Baker, Gero Lenhardt \\ Max-Planck-Institut für Bildungsforschung, Lentzeallee 94, D-1000 Berlin 33
}

\begin{abstract}
Zusam me nf assung: Der Beitrag untersucht ethnisch bedingte Unterschichtung der Berufspyramide und ethnische Diskriminierung in der beruflichen Statusallokation. Dazu werden Daten aus der Statistik der sozialversicherungspflichtig beschäftigten Arbeitnehmer und solche aus der Industriestatistik analysiert. Sie betreffen ausschließlich den Bereich männlicher vollzeitbeschäftigter Arbeiter. Eine Übersicht über einschlägige institutionelle Regelungen sowie eine Literaturübersicht erbringen zusätzliche Evidenz. Es ergeben sich nur schwache Hinweise auf ethnisch bedingte Unterschichtung und ethnische Diskriminierung in der Statusallokation. Der Ausländerstatus ist zwar ein Status minderen Rechts. Für diejenigen mit verfestigtem aufenthaltsrechtlichem und arbeitserlaubnisrechtlichem Status ergeben sich gegenüber Staatsbürgern aber nur geringe Nachteile.
\end{abstract}

\section{Vorbemerkung ${ }^{1}$}

Einwanderung, die Entstehung von Minderheiten und das berufliche Schichtensystem bilden einen sozialstrukturellen Zusammenhang, dem die Soziologie immer wieder Aufmerksamkeit zugewandt hat. Ein Aspekt dieses Themas ist die berufliche Statuszuweisung. Damit befaßt sich auch die Studie, von der hier berichtet wird. Sie bezieht sich auf die Bundesrepublik Deutschland und geht vor allem zwei miteinander verbundenen Fragen nach: (1) Werden die Individuen als Arbeitskräfte nach ihrer Nationalität im Statuszuweisungsproze $\beta$ unterschieden? (2) Entstehen in Verbindung mit der Immigration besondere Arbeitsverhältnisse oder werden die überkommenen unverändert auf die Neuankömmlinge ausgedehnt?

Die Integration des Arbeitsmarktes ist bedingt durch das Gewicht von zwei Normensyndromen, die in der BRD sowie in den meisten anderen Nationalstaaten auch anzutreffen sind: dem republikanischen Universalismus und dem Nationalismus. Sie sollen kurz bezeichnet werden. Dem schließt sich eine Skizee der Entwicklung der Ausländerpolitik an. Im Teil über Arbeitsmarktintegration werden die beiden genannten Dimensionen der Ungleichbehandlung dargelegt, sodann werden die Daten analysiert und anschließend diskutiert.

${ }^{1}$ Die Studie wurde unterstützt vom German Marshall Fund, Washington, D.C., und dem Max-Planck-Institut für Bildungsforschung, Berlin.

\section{Internationale Arbeitskräftemigration zwischen Republikanismus und Nationalismus}

Die soziale Identität der Individuen ist heute weltweit die von Staatsbürgern. Die Staatsbürgerrolle hat sich auf Kosten älterer Formen des Zusammenlebens durchgesetzt. Verbunden mit der Expansion des modernen Staates ist eine andere Entwicklung: Die Arbeitsteilung hat sich über Staatsgrenzen hinweg international intensiviert. Nicht nur Güteraustausch und Produktion, sondern auch die Arbeitsmärkte sind international geworden. Arbeitskräfteemigration ist ein weltweites Phänomen geworden (Sassen 1988). Daraus entsteht das Problem, wie Individuen, die zunehmend nationalstaatlich organisiert sind, in eine Wirtschaft integriert werden, die zunehmend international operiert. Die internationale Migration von Arbeitskräften und ihre gesellschaftliche Integration unterliegen vielfältigen Bedingungen. Darunter ist ein Widerspruch von Bedeutung, der die modernen Staaten kennzeichnet. Sie sind Nationalstaaten. Ihre Konstruktion bringt widersprüchliche Wertideen zum Ausdruck. Die modernen Staaten sind zum einen mit der Idee der Menschenrechte verbunden und zum anderen mit nationalistischen Vorstellungen. Die liberale Demokratie leitet die Rechte ihrer Bürger aus universalgültigen Menschenrechten ab. Dem entspricht die Vorstellung, daß prinzipiell alle Menschen Bürger einer Republik werden können, die sich zur Verfassung bekennen.

Im Gegensatz dazu legitimiert sich der Nationalismus aus der Vorstellung vom Bestehen einer besonderen nationalen Überlieferung. Sie steht im Gegensatz zu den Prinzipien des formalen Univer- 
salismus der Menschenrechte. In Deutschland hat diese Idee die Form des ethnischen Nationalismus. Ihm zufolge begründen Tradition und Blutsbande die soziale Integration. Die Stammesfremden, denen die unterstellte kulturelle Überlieferung fremd ist, gelten im naiven Bewußtsein als ,Barbaren', als ,unkultiviert" oder als moralisch minderwertig.

Die moderne Minderheitensoziologie, so hat Elias (1989: 177) beobachtet, entgeht nicht immer der Gefahr, nationalistische Stereotypen in wissenschaftlichem Gewand zu reproduzieren. Einwanderer werden zwar nicht als minderwertig porträtiert, unterstellter Tradition wegen wohl aber als Fremde, als unangepaßt, mit Sozialisationsdefiziten und abweichenden Wertorientierungen belastet. Diese Fremdheit gilt als Integrationsproblem und läßt die Frage nach Assimilation und entsprechenden erzieherischen Unternehmungen entstehen (vgl. dazu Esser/Friederich 1990). Für die soziale Konstruktion von Minderheiten und entsprechende Konflikte sind Kulturdifferenzen aber nur von zufälliger Bedeutung. Konflikte zwischen Deutschen und Türken zum Beispiel entzünden sich nicht an dem, worin sich beide unterscheiden, sondern an dem, worin sie sich gleichen: am Interesse an knappen Wohnungen und Arbeitsplätzen. Deswegen geht ein anderer Teil der Minderheitensoziologie davon aus, daß den Diskriminierenden die Eigenschaften der Diskriminierten gar nicht in den Blick geraten, daß Diskriminierung von Wahnsystemen oder Vorurteilen geleitet ist (vgl. dazu die Übersicht bei Heckmann 1981). Mit den modernen Nationalstaaten ist die Unterscheidung zwischen ,Eigenen' und ,Fremden' mit der Staatsbürgerschaft institutionalisiert und ist rechtlich geordnet. In Begriffen der Psychopathologie ist ihre Entwicklung dann nicht mehr zu begreifen (Lenhardt 1990).

Die vielfältigen Formen der Exklusion oder Inklusion von Ausländern lassen sich auf einem Kontinuum nationalistischer Exklusion anordnen. Dessen einen Pol bilden Verhältnisse radikalliberaler Demokratie. Den Gegenpol bilden Staaten, die zwischen Inländern und Ausländern radikal unterscheiden und im Extremfall ihre Grenzen gänzlich geschlossen halten. Wenn Zuwanderung nicht vermieden werden kann, dann kann der ausländischen Wohnbevölkerung die Teilhabe am gesellschaftlichen Leben partiell durch rechtliche oder andere Unterscheidungen verwehrt werden. Partielle Exklusion hat es in der Geschichte häufig gegeben. Wallerstein (1974) zeigt, daß sie für die ökonomische Expansion in den Randgebieten des Westens typisch gewesen ist.
Im Grundgesetz der Bundesrepublik Deutschland finden sich die Idee der Menschenrechte und nationalistische Wertideen nebeneinander. So werden die Rechte der Bürger im Artikel 1 mit der Würde des Menschen und nicht mit der Würde des Deutschen begründet; aber ein Teil der Freiheitsrechte ist nicht als Menschenrecht institutionalisiert, sondern an die Staatsbürgerschaft gebunden (Oberndörfer 1990). Die Ideen des völkischen Nationalismus zeigen sich auch in der gesetzlichen Definition dessen, was ein Deutscher ist. Deutscher ist nicht nur ein Bürger, der deutsche Staatsangehörigkeit, sondern auch derjenige, der deutsche Volkszugehörigkeit hat (Artikel 116 GG). Dem entspricht, daß im Staatsbürgerschaftsgesetz das ius soli nicht anzutreffen ist. Es verleiht in den westlichen Demokratien demjenigen ein Anrecht auf Staatsbürgerschaft, der im Hoheitsgebiet geboren ist.

\section{Aus Gastarbeitern werden Einwanderer}

\subsection{Kontrolle der Staatsgrenzen}

Der Gegensatz zwischen Nationalismus und dem Universalismus der Menschenrechte bestimmt auch die Einwanderung und die Arbeitsverhältnisse der sogenannten Gastarbeiter (Dohse 1981). Die kontrollierte Öffnung der Grenzen ging nicht auf eine Grundsatzentscheidung der Bundesregierung zurück, sondern war das Ergebnis von Einzelentscheidungen, die jede für sich als unbedeutend galten und nicht auf ihre langfristigen Folgen hin befragt wurden. Zusammengenommen aber resultierten sie in der millionenfachen Einwanderung ausländischer Arbeiter und ihrer Familien. Knappheit an Arbeitskräften, regional- und strukturpolitische Miseren, Inflation, die infolge steigender Löhne zu wachsen begonnen hatte, Gesichtspunkte der europäischen Integration usw., das waren die Probleme, angesichts derer die kontrollierte Öffnung der Grenzen als akzeptabel erschien. Diese heterogenen Entscheidungen lassen einen gemeinsamen Nenner erkennen: Gesichtspunkte nationaler Exklusion haben gegenüber anderen gesellschaftlichen Imperativen an Bedeutung verloren.

Die Ausländer, so hatte man aber geplant, sollten nicht als Bürger, sondern nur als Arbeitskräfte in Erscheinung treten, als ein Arbeitskräfteangebot, das sich an die Wechselfälle der Nachfrage anpassen ließe. Rechtsansprüche auf Aufenthalt und Arbeit sollten gering gehalten werden. Aber auch diese Ausgrenzung wurde in Frage gestellt. Zu- 
wanderungen konnten eingeschränkt, aber nicht gänzlich unterbunden werden. Abwanderungen hat man zu stimulieren versucht, aber die Mittel, die dazu von Gesetzes wegen und in der Öffentlichkeit als akzeptabel erschienen, reichten nicht allzu weit (Meier-Braun 1988: 64ff). Einer radikalen Repatriierungspolitik stehen vor allem folgende Umstände entgegen:

- Ein beträchtlicher Teil der ausländischen Wohnbevölkerung stammt aus den Staaten der Europäischen Gemeinschaft und genießt inzwischen das Recht auf Freizügigkeit.

- Die Nichteuropäer sind inzwischen durch Selbstbindungen der BRD geschützt. Ihr aufenthaltsrechtlicher und arbeitserlaubnisrechtlicher Status hat sich verfestigt.

- Übernationales Recht und zwischenstaatliche Vereinbarungen haben den Status der ausländischen Wohnbevölkerung ebenfalls verfestigt.

- In der westdeutschen Öffentlichkeit ist die fremdenfeindliche Schreckensherrschaft des Nationalsozialismus nicht vergessen. Liberale und Christen, Sozialdemokraten, Gewerkschaften und Wohlfahrtsverbände engagieren sich für die Rechte der Ausländer. Das gilt auch für die einschlägige Wissenschaft, die ein wichtiger Bestandteil der öffentlichen Meinung ist (Treibel 1988).

Diese Veränderungen spiegeln sich darin, daß im öffentlichen Bewußtsein aus ,Gastarbeitern' ,ausländische Mitbürger' geworden sind. Menschenrechtsgesichtspunkte haben an Bedeutung gewonnen.

\subsection{Inklusion und Exklusion der Gastarbeiter}

Der Widerspruch zwischen dem Universalismus der Menschenrechtsidee und dem nationalistischen Partikularismus kennzeichnet auch die soziale Lage derer, die sich dauerhaft in der BRD niedergelassen haben. Sie sind nicht Bürger der BRD geworden. Den ,Gastarbeitern' fällt es schwer, ihre alte Staatsbürgerschaft aufzugeben, und den Sachwaltern des ethnischen Nationalismus in Deutschland, sie einzubürgern. $\mathrm{Da}$ der einzelne über seine Staatsangehörigkeit nach Gesichtspunkten der Zweckmäßigkeit befinden kann, ist eine Vorstellung, die nationalen Sentiments widerspricht.

Die Einwanderer sind Ausländer geblieben, und das ist gleichbedeutend damit, da $B$ sie in einem Status minderen Rechts leben. Dabei leben die verschiedenen Kategorien der Ausländer keines- wegs in einem einheitlichen Rechtsstatus. Je nach Herkunftsland und Aufenthaltsdauer variieren ihre Rechtspositionen. Aber gemeinsam ist allen, $\mathrm{da} B$ sie in den drei Rollen unterprivilegiert sind, die in den westlichen Gesellschaften die individuelle Existenz strukturieren: nämlich als Staatsbürger, in der Rolle des Klienten gegenüber dem Sozialstaat und schließlich im Hinblick auf gesellschaftliche Teilhaberechte, vor allem auf dem Arbeitsmarkt (Marshall 1965).

In der nationalistisch inspirierten Diskriminierung ausländischer Arbeitskräfte scheint in moderner Form ein Problem fortzuleben, das im 18. und 19. Jahrhundert Gegenstand heftiger sozialer Konflikte in den westlichen Gesellschaften war, nämlich „die Erweiterung der Staatsbürgerschaft auf die unteren Klassen" (Bendix 1982). Sie ist im Falle der ausländischen Arbeiter nicht gelungen, sie ist jedoch vorangekommen. Die Staatsangehörigkeit, so beobachtet Sieveking (1990), verliert ihre überkommene Bedeutung als Instrument politischer Steuerung.

\section{Arbeitsmarktintegration}

Die Frage nach Diskriminierung und Integration auf dem Arbeitsmarkt soll in zweierlei Richtung verfolgt werden (vgl. dazu Baker/Lenhardt 1990). Zum einen soll die berufliche Statuszuweisung untersucht werden und zum andern die Konstruktion der beruflichen Schichtenpyramide. Wird die Angebotsseite des Arbeitsmarktes nach ethnischen Kriterien unterschieden, dann zeigt sich das in der beruflichen Statusallokation. Aber auch in der Statuskonstruktion, das heißt auf der Nachfrageseite, können sich ethnische Kategorien herausbilden. Diese Unterscheidung wird im folgenden erläutert.

a) Die Frage nach der Bedeutung ethnischer Merkmale in der Statusallokation hat in der Soziologie die größte Aufmerksamkeit gefunden. Hier geht es darum, ob bei der Vergabe von Arbeitsplätzen zwischen inländischen und ausländischen Arbeitskräften, also nach einem leistungsfremden Kriterium, unterschieden wird mit der Folge, daß den einen der Zugang zu bestimmten Bereichen des beruflichen Schichtensystems gänzlich verschlossen bleibt oder erschwert wird. Dabei wird das System der Berufspositionen als gegeben unterstellt. Es geht allein darum, ob die Ausländereigenschaft zum Ansatz für spezifische Allokationsprozesse wird.

Dem entspricht die verbreitete Annahme, die Ausländerbeschäftigung habe Deutschen besondere 
Karrierevorteile verschafft. Eine Anzahl von ihnen sei aus Gründen ethnischer Privilegierung gewissermaßen auf dem Rücken ihrer ausländischen Kollegen aufgestiegen. Die Einwanderer hätten anstelle der aufgestiegenen Deutschen die untersten Plätze der Berufshierarchie übernommen, und ein ethnozentrisches Ressentiment halte sie dort fest. $\mathrm{Da} \beta$ ausländische Arbeitskräfte im Vergleich mit deutschen häufiger und länger von $\mathrm{Ar}$ beitslosigkeit betroffen sind, weniger verdienen und seltener Karriere machen, höheren Unfallrisiken unterliegen, häufiger im Akkord und im Schichtbetrieb arbeiten, weniger qualifizierte, aber belastendere Arbeiten verrichten müssen usw., kann als gut gesichert gelten. Im Vergleich mit deutschen Arbeitnehmern haben die ausländischen in allen Dimensionen der Berufsrolle schlechtere Bedingungen (vgl. dazu Kühne/Schäfer 1986; Gillmeister/Kurthen/Fijalkowski 1989; Bielefeld 1989; Reimann/Reimann 1987; Zapf/Brachtl 1984; Szydlik 1990).

$\mathrm{Da}$ diese Differenzen auf ethnische Diskriminierung zurückgehen, darf aber nicht ungeprüft unterstellt werden. Hier können auch andere Faktoren eine Rolle spielen. Von größtem Einfluß auf Karrierechancen sind die Wirkungen des schulischen Berechtigungswesens; wer erst jenseits des Kindheits- und Jugendalters in die BRD eingewandert ist, konnte die notwendigen Zeugnisse deutscher Schulen nicht erwerben und geriet deswegen in eine schwierige Situation auf dem Arbeitsmarkt (vgl. Baker/Lenhardt 1988). In Rechnung zu stellen sind Beeinträchtigungen der Karrierechancen infolge mangelnder Sprachkenntnisse oder mangelnder Vertrautheit mit den Lebensverhältnissen in der BRD. Auch Anciennitäts- und Senioritätsregeln benachteiligen Neuankömmlinge und Jüngere unabhängig von irgendwelchen ethnischen Merkmalen. Daß Zuwanderer auch im Fall ethnischer Gleicheit noch über sehr lange Zeiträume und über Generationen hinweg mit Karrierenachteilen zu kämpfen haben, hat Lüttinger in einer Untersuchung zur Integration deutscher Vertriebener und Flüchtlinge gezeigt. „Die erfolgreiche Eingliederung wird erst bei den Kindern und Kindeskindern der Zuwanderer in vollem Umfang erreicht. Geringere schulische und berufliche Chancen und ein selektiver Strukturwandel vermitteln das Bild einer teilweise ,segregierten' gesellschaftlichen Entwicklung.“ (Lüttinger 1986: 35)

b) Ethnische Kategorien können sich auch in der Struktur der Berufspyramide herausbilden. Besonders sinnfällige Beispiele dafür bilden Sklaverei oder Zwangsarbeit, zu der Millionen deportierter Menschen während des Nationalsozialismus gezwungen wurden. Diese Arbeitsverhältnisse enthalten Regelungen, die sich als bloße Verstöße gegen das Leistungsprinzip nicht angemessen beschreiben lassen. Merkmale der Rasse oder nationalen Zugehörigkeit werden hier zum Ansatzpunkt für die Konstruktion ganz andersartiger Formen der Arbeitsteilung und Eigentumsverhältnisse mit spezifischen Formen der Machtausübung und Chancen, sich dagegen zu wehren. Ethnische Kategorienbildung in der Konstruktion des beruflichen Schichtensystems manifestiert sich also darin, daß Arbeitsverhältnisse entstehen, mit deren Existenz unter Bedingungen formaler Gleichbehandlung der Arbeitskräfte nicht zu rechnen ist. Man spricht dann von Unterschichtung. Eine weniger radikale Form der Unterschichtung ist lediglich quantitativer Natur. Hier nimmt der Anteil der ungünstigeren, zum Beispiel schlecht entlohnten oder taylorisierten Arbeiten aus Gründen ethnischer Diskriminierung zu, während die institutionellen Regelungen der Arbeitswelt im übrigen unverändert bleiben. Mit dieser Form der Unterschichtung befaßt sich die Studie von HoffmannNowotny (1973). In der ausländerpolitischen Debatte in Deutschland entspricht dem die These, $\mathrm{daB}$ die Einwanderung zu einer Unterschichtung der bis dahin bestehenden Berufsordnung geführt hätte oder wenigstens zu einer Verbreiterung ihres Sockels.

Für die Annahme nationalistischer Diskriminierung haben Dombois (1980) und Dohse (1981) Überlegungen geltend gemacht, die sowohl die These von den Karrierevorteilen Deutscher wie die Unterschichtungshypothese begründen können: Wegen ihres schwachen aufenthaltsrechtlichen und arbeitserlaubnisrechtlichen Status und wegen ethnozentrischer Vorurteile in der deutschen Arbeitnehmerschaft sind die ausländischen Arbeitskräfte gegenüber der betrieblichen Personalpolitik in einer schwachen Position. Ihre Belange scheinen gelegentlich auch von der betrieblichen Interessenvertretung mit geringerem Nachdruck vertreten zu werden (vgl. dazu auch Gillmeister/Kurthen/Fijalkowski 1989). Diese Umstände legen es den Betrieben nahe, belastende Arbeitsplätze und das Risiko der Arbeitslosigkeit vor allem ausländischen Arbeitskräften zuzudiktieren. Deren Ausgrenzung reduziert die Risiken für den Betriebsfrieden, die repressive Arbeitsverhältnisse in sich bergen. Eine schwache Verhandlungsposition der Arbeitnehmerschaft oder Teilen davon erlaubt es den Betrieben auch, gegebenenfalls 
rücksichtsloser mit den Beschäftigten zu verfahren und Arbeitsbedingungen zu schaffen, die bei anderen Machtverhältnissen nicht durchzusetzen wären.

\section{Daten}

$\mathrm{Zu}$ beiden Dimensionen möglicher Diskriminierung werden im folgenden Berechnungen dargelegt. Sie betreffen zunächst die Statuskonstruktion, zielen also auf die Frage, ob Ausländerbeschäftigung zu Unterschichtungsphänomenen geführt hat. Sodann wird nach ethnischer Diskriminierung in der Statusallokation gefragt, wobei die Berufspyramide als stabil unterstellt wird.

(1) Unterschichtung soll hier in zweierlei Weise indiziert werden. Sie müßte sich zum einen als Zuwachs der ungelernten beziehungsweise angelernten Arbeit im Vergleich zur Facharbeit zeigen. Zum andern läßt sie sich indizieren durch Veränderungen der Löhne. Deswegen wird der Einfluß der Ausländerbeschäftigung auf die Qualifikationsstruktur und auf Bruttostundenverdienste geprüft.

Diese beiden Indikatoren erfassen freilich nur zwei von verschiedenen möglichen Unterschichtungsphänomenen. Illegale Formen der Beschäftigung, die vielleicht radikalste Form der Unterschichtung, kann so überhaupt nicht sichtbar werden. Unsichtbar bleiben ebenso Veränderungen innerhalb der Kategorie nichtgelernter Arbeit. Außer Betracht bleibt schließlich die Entwicklung der sogenannten ungeschützten Arbeitsverhältnisse. Eine Expansion der ungünstigeren legalen Arbeitsverhältnisse bildet allerdings die wahrscheinlichste Form der Unterschichtung. Weil sie das normative Gefüge des Arbeitsmarkts am wenigsten beeinträchtigt, ist sie am leichtesten durchzusetzen. Im Auge zu behalten ist freilich auch, daß Unterschichtung nicht unter allen Umständen auf Diskriminierung hindeuten muß. Das ist weiter unten zu erörtern.

(2) Auf ethnische Diskriminierung in der Statusallokation werfen Daten zur beruflichen Mobilität ein Licht. Es wird untersucht, inwieweit die ausländischen Arbeitskräfte in der untersten beruflichen Schicht verblieben sind und inwieweit ihnen beruflicher Aufstieg gelungen ist. Geprüft wird weiterhin, ob die Ausländerbeschäftigung unter den deutschen Arbeitskräften einen Zuwachs an beruflichem Aufstieg bewirkt hat.

(3) Als ein Hinweis auf ethnische Diskriminierung in der Statusallokation kann auch die Verteilung der ausländischen Arbeitskräfte über die verschiedenen Wirtschaftszweige angesehen werden. So wird geprüft, ob Ausländer anders als deutsche Arbeitskräfte über den Arbeitsmarkt verteilt und in Wirtschaftszweigen mit besonders belastender Arbeit konzentriert sind.

In allen diesen Untersuchungen berücksichtigen wir ausschließlich vollzeitbeschäftigte männliche Arbeiter. Die Verhältnisse auf dem Arbeitsmarkt sind nicht einheitlich, deswegen ist die Unterscheidung verschiedener Sektoren geboten. Zeit- und Platzgründe machen die Reduktion der Studie auf nur einen Arbeitsmarktsektor notwendig. Aus verschiedenen Gründen empfiehlt sich dabei die Konzentration auf männliche Vollzeitbeschäftigte. Männliche Vollzeitarbeiter bilden die größte Beschäftigtengruppe unter den Ausländern. Als Vollzeitbeschäftigte repräsentieren sie zentrale Bereiche des Arbeitsmarktes mit großer institutioneller Regelungsdichte. Normen zur Unterscheidung deutscher und nichtdeutscher Arbeitskräfte müßten deswegen vor allem in diesem Bereich anzutreffen sein. Die Konzentration auf Männer schließt Einflüsse geschlechtsspezifischer Diskriminierung aus, deren Bedeutung oft hervorgehoben worden ist. Darum soll es in dieser Studie aber nicht gehen. Es kann jedoch mitgeteilt werden, daß sich ganz ähnliche Befunde wie die im folgenden berichteten ergeben, wenn man die ausländische Arbeitnehmerschaft insgesamt in die Gleichungen einbezieht.

Die Analysen stützen sich auf Daten, die aus der Statistik der sozialversicherungspflichtig beschäftigten Arbeitnehmer und aus der Industriestatistik gewonnen wurden (Statistisches Bundesamt $1977 \mathrm{ff}$ (a) und (b)). In der Statistik der sozialversicherungspflichtig beschäftigten Arbeitnehmer sind vor allem die Angaben zur ,Stellung im Beruf' bedeutsam. Jeder Beschäftigte und damit jeder Arbeitsplatz wird entsprechend klassifiziert. Bei ,Stellung im Beruf werden unterschieden: ungelernte und angelernte Arbeiter, hier als Nichtgelernte zusammengefaßt, sodann Facharbeiter und Vorarbeiter, Meister und schließlich Angestellte. Jedermann wird darüber hinaus kategorisiert unter anderem nach Geschlecht, Nationalität sowie auch dem Wirtschaftszweig und dem Ort, an dem er beschäftigt ist.

Aus diesen Angaben haben wir durch Aggregation der Individualdaten Untersuchungseinheiten konstruiert, nämlich Landeswirtschaftszweige. Ihre Zahl ergibt sich aus der Anzahl der Bundesländer und der Wirtschaftszweige, die in der Statistik 
unterschieden werden. Wir haben jeweils Schleswig-Holstein und Hamburg, Bremen und Niedersachsen, Rheinland-Pfalz und das Saarland zusammengefaßt; Bayern wurde unterteilt in Nord- und Südbayern. Hinzu kommen Nordrhein-Westfalen, Hessen, Baden-Württemberg und Berlin. Die Statistik unterscheidet 94 Wirtschaftszweige; sie verteilen sich auf die vier Wirtschaftsbereiche Landund Forstwirtschaft, Tierhaltung und Fischerei (I), Produzierendes Gewerbe (II), Handel und Verkehr (III), Sonstiges (IV). Da nicht alle Wirtschaftszweige in allen unseren Regionen - im folgenden kurz als Länder bezeichnet - vertreten sind, ergibt sich als Summe der Landeswirtschaftszweige, die unsere Untersuchungseinheiten bilden, die Zahl 832.

Jeder Landeswirtschaftszweig läßt sich mit den Angaben zu ,Stellung im Beruf' als Schichtenpyramide beschreiben, und für jeden liegen Angaben zum Anteil der ausländischen Arbeitskräfte vor. So kann der Einfluß der Ausländerbeschäftigung auf die Struktur dieser Einheiten und damit des westdeutschen Arbeitsmarktes überprüft werden. Mit Ausnahme der Selbständigen und Beamten wird so der gesamte Arbeitsmarkt erfaßt. Die Dekade von 1977 bis 1986 bildet den Untersuchungszeitraum; wir haben ihn mit den Daten der Jahre 1977, 1981 und 1986 beschrieben.

In analoger Weise wurden Daten aus der Industriestatistik benutzt. Die Industriestatistik liefert unter anderem Angaben über die gezahlten Bruttostundenverdienste in den einzelnen Wirtschaftszweigen der verschiedenen Bundesländer, aber nur für die industriellen. Dabei werden drei Leistungsgruppen unterschieden. Sie bilden ebenfalls eine Hierarchie, wobei dem Merkmal Qualifikation wiederum besonderes Gewicht zukommt. Mit dem Qualifikationssystem der ,Stellung im Beruf' ist diejenige nach Leistungsgruppen aber nicht identisch.

Die Industriestatistik unterscheidet zwischen Bruttostundenverdiensten, die für Männer und Frauen bezahlt werden, aber nicht zwischen denen für Ausländer und Inländer. Man mag das als ersten Hinweis darauf deuten, daß nationale Zugehörigkeit - so wie in der Statistik - auch im industriellen Alltag nur von eingeschränkter Bedeutung ist. Für unsere Studie ergibt sich daraus aber die Notwendigkeit, Ausländeranteile aus der Statistik der sozialversicherungspflichtig Beschäftigten zu gewinnen und auf die Einheiten zu übertragen, die aus der Industriestatistik konstruiert wurden. Das ist nicht unproblematisch und mahnt zur Vor- sicht im Umgang mit den Ergebnissen. Da es uns aber nur um Trendaussagen geht, kann man diese Unsicherheit in Kauf nehmen.

Die Industriestatistik gibt, wie schon betont, Auskunft nur über einen Sektor des Arbeitsmarktes, den industriellen. Bekanntlich sind hier aber die meisten Ausländer beschäftigt, und deswegen müßten Effekte der Ausländerbeschäftigung hier auch geballt und mithin besonders deutlich hervortreten. Wenn Ausländerbeschäftigung in Unterschichtung resultierte, dann müßte sie sich in einer Verringerung der Löhne zeigen, vor allem derjenigen in der niedrigsten Leistungsgruppe, der dritten. Wir haben die Bruttostundenverdienste jeweils vom Oktober der drei ausgewählten Jahre unseres Untersuchungszeitraums herangezogen. Sie wurden auf der Basis von 1980 standardisiert, um inflationsbedingte Veränderungen auszuschalten.

Den Einfluß der Ausländerbeschäftigung auf das berufliche Schichtensystem messen wir mit Standard-Querschnitts-OLS-Regressionen. Die Zahl vollzeitbeschäftigter ausländischer männlicher Arbeiter bildet dabei die unabhängige Variable und der Quotient aller männlichen vollzeitbeschäftigten Facharbeiter zu allen männlichen vollzeitbeschäftigten nichtgelernten Arbeitern, also Deutsche und Ausländer zusammengenommen, die abhängige Variable. Kontrolliert wird der Einfluß von vier Variablen.

Zum einen ist der mögliche Einfluß der Zahl der Beschäftigten in einer Untersuchungseinheit (GröBe) zu kontrollieren sowie deren Veränderungen (Wachstums-Größe 1977). Zum anderen wird kontrolliert, ob der Wirtschaftszweig dem privaten oder öffentlichen Sektor angehört (Sektor, Privat $=1$, Öfentlich $=0$, der Industrie oder anderen Wirtschaftsbranchen (Branche: Industrie $=1$, Andere $=0$ ).

In Rechnung zu stellen ist darüber hinaus das Folgende. Die Ratio gelernter und nichtgelernter Arbeiter kann auch durch die vorherige Qualifikationsstruktur in einem Landeswirtschaftszweig bestimmt sein. Es ist auch denkbar, daß diese Ratio wegen organisatorischer und institutioneller Rigidität nur mit Verzögerung auf Effekte der Ausländerbeschäftigung reagiert. Um das zu berücksichtigen, berechnen wir ein geometrisch verteiltes lag model (Kmenta 1971). Die allgemeine Formel des lag model lautet:

(1) $Y_{t}=\alpha+\beta_{0} X_{t}+\beta 1 X_{t-1}+\beta 2 X_{t-2}+\ldots$ $+\beta m X_{t-x}+\varepsilon_{t}$. 
Diese Gleichung enthält aber ein Problem. Die Multicollinearität gegenwärtiger und früherer Werte von X (Umfang der Ausländerbeschäftigung), mit der mit Sicherheit zu rechnen ist, steht exakten Schätzungen der Standardabweichungen der Koeffizienten im Wege. Geht man davon aus, $\mathrm{da} B$ frühere Werte von $X$ die Anpassung gegenwärtiger Werte von $\mathrm{Y}$ in geringerem Umfang tangieren, dann läßt sich ein geometrisches lag model benutzen, um ähnliche Effekte wie in (1) zu berechnen. Der Ausdruck dafür lautet:

(2) $Y_{t}=\alpha+\beta_{o}\left(X_{t}+\Gamma X_{t-1}+\Gamma^{2} X_{t-2}+\ldots.\right)+\varepsilon_{t}$, wenn $0 \leq \Gamma \geq 1$.

Die Gleichung (2) kann transformiert werden in eine Form, die sich so berechnen läßt:

(3) $Y_{t}=\alpha_{0}+\beta_{0} X t+\Gamma Y_{t-1}+\varepsilon^{\prime}{ }^{2}$

Wenn die Gleichung (3) gerechnet ist, ergibt sich für die Berechnung der Anpassungseffekte:

$\beta=\beta_{\mathrm{o}} /(1-\Gamma)$

$\alpha=\alpha_{o} /(1-\Gamma)$

\section{Ergebnisse}

Die Tabelle 1 enthält fünf Gleichungen zur ersten Hypothese. Sie betreffen den Einfluß der Ausländerbeschäftigung auf die Form des beruflichen Schichtensystems definiert durch das Merkmal ,Stellung im Beruf'. Abhängige Variable ist das Verhältnis zwischen allen männlichen vollzeitbeschäftigten Facharbeitern zu allen vollzeitbeschäftigten nichtgelernten männlichen Arbeitern in einem Landeswirtschaftszweig. Ausländer und Inländer sind dabei zusammengenommen. Der Durchschnittswert für diesen Quotienten beträgt 2.1 Facharbeiter für jeden Nichtgelernten. Falls die Unterschichtungshypothese zutrifft, müßte dieser Quotient in den Einheiten mit größerer Ausländerbeschäftigung kleiner sein.

${ }^{2}$ Gleichung (2) wurde so umgeformt, daß ihre Schätzung durch eine zweimalige sukzessive Anwendung der Koyck-Transformation möglich ist (Kmenta 1977: 478). Da bei Gleichung (3) die Annahme unkorrelierter Fehler verletzt ist $\left(Y_{t}\right.$ und $Y_{t-1}$ haben eine gemeinsame Fehlerkomponente), verwenden wir eine OLS-Regression, in der $\mathrm{p}$ (Autokorrelation erster Ordnung) nach der Cochrane-Orcutt-Methode geschätzt wurde (Hanushek/Jackson 1977).
Die ersten drei Gleichungen sind OLS-Schätzungen von Querschnittseffekten zu Beginn (1977), in der Mitte (1981) und am Ende (1986) der Untersuchungsperiode. Die Gleichungen zeigen im wesentlichen dasselbe Ergebnis. Der ersten Hypothese entsprechend geht umfangreichere Ausländerbeschäftigung mit einer kleineren Ratio von Facharbeitern zu nichtgelernten Arbeitern einher. Unübersehbar ist jedoch, daß die Effekte sehr klein sind. Im Jahr 1986 zum Beispiel, in dem dieser Effekt am stärksten in Erscheinung tritt, verringert die Anwesenheit einer durchschnittlichen Zahl ausländischer Arbeiter den Quotienten nur um 0.15, also nur um sieben Prozent. Und eine zehnprozentige Zunahme der durchschnittlichen Zahl männlicher Ausländer reduziert diesen Quotienten nur um ein weiteres Prozent auf sechs Prozent.

Die beiden letzten Gleichungen in Tabelle 1 sind gewogene Schätzungen eines distributed lag model. Dabei ist unterstellt, daß sich der Quotient gelernter zu nichtgelernter Arbeiter nur mit Verzögerung an frühere Werte der Ausländerbeschäftigung anpaßt. Der ersten Hypothese zufolge müßte die Ausländerbeschäftigung diesen Quotienten verringern. Die Berechnung der Anpassungseffekte in den Gleichungen $E$ und $D$ ergibt:

D. $1981-1986$ Quotient $=3.89-2.73^{-04}$ (Anzahl der ausländischen männlichen Arbeiter)

E. $1977-1986$ Quotient $=2.27-1.55^{-04}$ (Anzahl der ausländischen männlichen Arbeiter)

In beiden Fällen nimmt der Quotient gelernter zu nichtgelernten Arbeitern infolge der Ausländerbeschäftigung $a b$. Aber auch hier sind die Effekte sehr klein. So beträgt die Abnahme der Quotienten zwischen 1977 und 1986 in einem Landeswirtschaftszweig mit einer durchschnittlichen Zahl ausländischer Arbeiter zum Beispiel nur -0.12 oder fünf Prozent des Quotienten.

Nach den Berechnungen ethnischer Diskriminierung in der Konstruktion des beruflichen Schichtensystems sollen jetzt Daten dargelegt werden, die ethnische Diskriminierung in sozialen Aufstiegsprozessen betreffen. Ethnische Diskriminierung müßte sich darin zeigen, daß ausländischen Arbeitskräften berufliche Karrieren versperrt sind, daß sie also in der untersten Schichtenkategorie verbleiben und nicht wie ihre deutschen Kollegen aufsteigen. Ohne Längsschnittdaten zu individuellen Karriereverläufen ist diese Frage schwer zu überprüfen. Man kann jedoch mit den hier verfügbaren Daten aggregierte Veränderungen der ausländischen Arbeitskräfte in den Schichtenkatego- 
Tabelle 1 Ausländerbeschäftigung und berufliche Schichtung: Zusammenhänge zwischen der Zahl vollzeitbeschäftigter ausländischer Arbeiter (Männer) und dem Quotienten von allen (deutschen und ausländischen) Facharbeitern und nichtgelernten Arbeitern in den Länderwirtschaftszweigen $(N=832)^{\prime}$

\begin{tabular}{|c|c|c|c|c|c|c|c|c|}
\hline & \multicolumn{6}{|c|}{$\begin{array}{l}\text { Unabhängige } \\
\text { Variablen }\end{array}$} & \multirow[t]{2}{*}{ Konstante } & \multirow[t]{2}{*}{$R$} \\
\hline & $\begin{array}{c}\text { Ausl. } \\
\text { Arbeiter }\end{array}$ & Lag Term & Größe & Wachstum & Sektor & Branche & & \\
\hline a. 1977 & $\begin{array}{l}-1.01^{-04} \\
\left(9.02^{-05}\right)\end{array}$ & & $\begin{array}{l}-2.57^{-06} \\
\left(9.22^{-06}\right)\end{array}$ & $\begin{array}{c}3.12^{-06} \\
\left(3.28^{-06}\right)\end{array}$ & $\begin{array}{c}-.15 \\
(1.12)\end{array}$ & $\begin{array}{l}-.76^{* *} \\
(.31)\end{array}$ & $\begin{array}{l}2.55^{* *} \\
(.21)\end{array}$ & $.12^{* *}$ \\
\hline b. 1981 & $\begin{array}{l}-1.46^{-04 *} \\
\left(6.65^{-05}\right)\end{array}$ & & $\begin{array}{l}-5.71^{-06} \\
\left(1.13^{-06}\right)\end{array}$ & $\begin{array}{l}-4.64^{-06} \\
\left(1.03^{-06}\right)\end{array}$ & $\begin{array}{l}-.83 \\
(.75)\end{array}$ & $\begin{array}{l}-.59 \\
(.25)\end{array}$ & $\begin{array}{l}2.32^{* *} \\
(.15)\end{array}$ & $.14^{*}$ \\
\hline c. 1986 & $\begin{array}{l}-2.00^{-04 *} \\
\left(8.47^{-05}\right)\end{array}$ & & $\begin{array}{c}1.02^{-05} \\
\left(5.44^{-06}\right)\end{array}$ & $\begin{array}{l}-1.79^{-04 *} \\
\left(7.75^{-05}\right)\end{array}$ & $\begin{array}{l}.09 \\
(.75)\end{array}$ & $\begin{array}{l}-.50^{*} \\
(.23)\end{array}$ & $\begin{array}{l}2.40^{* *} \\
(.15)\end{array}$ & $.16^{* *}$ \\
\hline d. $1981-1986$ & $\begin{array}{l}-2.46^{-05} \\
\left(2.95^{-05}\right)\end{array}$ & $\begin{array}{l}.91^{* *} \\
(.02)\end{array}$ & & & & & $\begin{array}{l}.35^{* *} \\
(.07)\end{array}$ & $.86^{* *}$ \\
\hline e. $1977-1986$ & $\begin{array}{c}-7.89^{-05 *} \\
\left(4.26^{-05}\right)\end{array}$ & $\begin{array}{l}.49^{* *} \\
(.02)\end{array}$ & & & & & $\begin{array}{l}1.16^{* *} \\
(.10)\end{array}$ & $.68^{* *}$ \\
\hline
\end{tabular}

* $\mathrm{p}<.05$

** $\mathrm{p}<.01$

1 Die Gleichungen a-c stellen OLS metrische Koeffizienten dar (Standardabweichungen). Die Gleichungen d und e sind gewichtete OLS metrische Koeffizienten (Standardabweichungen).

Tabelle 2 Männliche vollzeitbeschäftigte Arbeiter nach .Stellung im Beruf“, 1977 und 1986

\begin{tabular}{lc}
\hline Stellung im Beruf & Veränderung in Prozent \\
\hline Meister & 18,4 \\
Facharbeiter & $-13,0$ \\
Arbeiter & \\
(ungelernte und angelernte) & $-22,3$ \\
Zusammen & $-19,1$ \\
\hline
\end{tabular}

rien auf Aufstiegsprozesse hin befragen. Da die Einwanderung inzwischen erheblich reduziert wurde, hat sich die ausländische Erwerbsbevölkerung stabilisiert. Man erkennt das an ihrem wachsenden Durchschnittsalter (vgl. dazu Trommer 1991). Dreierlei könnte erwartet werden: 1 . beruflicher Aufstieg, 2. Immobilität und 3. Ausscheiden aus dem Arbeitsmarkt und zum Beispiel Rückkehr ins Herkunftsland.

Die Tabelle 2 zeigt die Verteilung der Einwanderer über die Kategorien der ,Stellung im Beruf' im Jahr 1977 und 1986. Die Daten lassen zweierlei erkennen:

Die Zahl der ausländischen Beschäftigten sinkt. Am stärksten schrumpft dabei die unterste Schich- tenkategorie. Ein Teil ist arbeitslos und in der Bundesrepublik verblieben. Andere sind in der stillen Reserve oder nach Hause zurückgekehrt, sei es, weil ihre Karriereerwartungen enttäuscht wurden, sei es, weil sie ihre mit der Gastarbeiterexistenz verbundenen Lebensziele erreicht hatten und für eine Rückkehr votierten.

Es gibt aber auch Aufstiegsprozesse. Es zeigt sich, daß die oberste Kategorie, die der Meister, wächst. $\mathrm{Zu}$ beobachten ist ebenfalls eine Zunahme derjenigen ausländischen Arbeitskräfte, die im Angestelltenverhältnis tätig sind. Im Falle der Meister kann auf sozialen Aufstieg geschlossen werden, im Falle der Angestellten ist ein solcher Schluß jedoch problematisch. Denn eine Reihe 
von Angestelltentätigkeiten rangiert auf einem Schichtenniveau, das unter demjenigen von Facharbeitern liegt. Bemerkenswert ist auch, daß die Kategorie Facharbeiter ausländische Arbeitskräfte verliert. Der relative Verlust ist aber kleiner als derjenige an ausländischen Arbeitskräften aller Qualifikationskategorien.

Es ist schwierig, von diesen Daten auf Diskriminierung zu schließen. Daß die Zahl ausländischer Meister zunimmt, deutet auf Aufstiegsmobilität hin. Welche Umstände sich dagegen in der Reduktion der Facharbeiter- und Nichtgelerntenkategorien manifestieren, ist offen. Von dem im Vergleich zu Deutschen häufigeren Ausscheiden der Ausländer aus der Erwerbstätigkeit kann nicht einfach auf Diskriminierung geschlossen werden, auch dann nicht, wenn dieses Ausscheiden unfreiwillig war. Dies wird im folgenden noch diskutiert.

Zunächst sollen Daten zu einer Frage dargelegt werden, die in der ausländerpolitischen Diskussion große Aufmerksamkeit gefunden hat. Vorbehalte gegenüber der Ausländerbeschäftigung speisen sich häufig aus der Befürchtung, die mit der $\mathrm{Zu}$ wanderung verbundene Erweiterung des Arbeitskräfteangebots verschärfe die Konkurrenz auf dem Arbeitsmarkt. Daraus ergäbe sich eine Ver- schlechterung der Arbeitsbedingungen auch der deutschen Arbeitnehmer. Unterstellt ist bei dieser Annahme, daß der Arbeitsmarkt wirklich Markt ist, daß hier Konkurrenzverhältnisse herrschen und daß folglich Deutsche und ausländische Arbeitskräfte austauschbar, das heißt gleich sind. Eine Verschlechterung der Berufschancen deutscher Arbeitnehmer infolge verschärfter Konkurrenz lieBe folglich auf formale Gleichbehandlung und mithin auf Integration der Ausländer auf dem Arbeitsmarkt schließen.

Tabelle 3 zeigt, wie sich die Ausländerbeschäftigung auf die Ratio von deutschen Facharbeitern und deutschen nichtgelernten Arbeitern auswirkt. Dabei geht es wieder ausschließlich um männliche Vollzeitbeschäftigte. Eine Verschlechterung der Arbeitsbedingungen deutscher Arbeitnehmer müßte sich in einer Verkleinerung des Quotienten zeigen. Kontrolliert werden dieselben vier Variablen wie oben, und berechnet werden Querschnittseffekte zu den gleichen Zeitpunkten und zwei distributed lag models. Die Ergebnisse aller fünf Gleichungen stimmen überein: ausländische männliche Vollzeitarbeiter verringern den Quotienten deutscher Facharbeiter und deutscher nichtgelernter Arbeiter. Sie deuten also auf Statuseinbußen deutscher Arbeitnehmer und mithin auf

Tabelle 3 Ausländerbeschäftigung und die Stellung Deutscher im Beruf: Zusammenhänge zwischen der Anzahl vollzeitbeschäftigter ausländischer Arbeiter (Männer) und dem Quotienten deutscher Facharbeiter und Nichtgelernter in den Länderwirtschaftszweigen $(N=832)^{\prime}$

\begin{tabular}{|c|c|c|c|c|c|c|c|c|}
\hline & \multicolumn{6}{|c|}{$\begin{array}{l}\text { Unabhängige } \\
\text { Variablen }\end{array}$} & \multirow[t]{2}{*}{ Konstante } & \multirow[t]{2}{*}{$\mathbf{R}$} \\
\hline & $\begin{array}{c}\text { Ausl. } \\
\text { Arbeiter }\end{array}$ & Lag Term & Größe & Wachstum & Sektor & Branche & & \\
\hline a. 1977 & $\begin{array}{l}-9.72^{-05} \\
\left(9.44^{-05}\right)\end{array}$ & & $\begin{array}{c}-9.60^{-07} \\
\left(9.69^{-06}\right)\end{array}$ & $\begin{array}{c}2.08^{-06} \\
\left(3.46^{-06}\right)\end{array}$ & $\begin{array}{c}-.12 \\
(1.19)\end{array}$ & $\begin{array}{l}-.66^{*} \\
(.33)\end{array}$ & $\begin{array}{l}2.8^{* *} \\
(.22)\end{array}$ & $.10^{*}$ \\
\hline b. 1981 & $\begin{array}{c}-1.20^{-04} \\
\left(7.25^{-05}\right)\end{array}$ & & $\begin{array}{c}3.73^{-06} \\
\left(7.38^{-06}\right)\end{array}$ & $\begin{array}{c}7.64^{-07} \\
\left(2.60^{-06}\right)\end{array}$ & $\begin{array}{l}-.82 \\
(.93)\end{array}$ & $\begin{array}{l}-.47 \\
(.26)\end{array}$ & $\begin{array}{l}2.6^{* *} \\
(.18)\end{array}$ & .11 \\
\hline c. 1986 & $\begin{array}{l}-2.16^{-04 *} \\
\left(8.95^{-05}\right)\end{array}$ & & $\begin{array}{c}5.66^{-06} \\
\left(7.24^{-06}\right)\end{array}$ & $\begin{array}{c}1.85^{-06} \\
\left(2.48^{-06}\right)\end{array}$ & $\begin{array}{c}.53 \\
(.85)\end{array}$ & $\begin{array}{l}-.41 \\
(.23)\end{array}$ & $\begin{array}{l}2.6^{* *} \\
(.16)\end{array}$ & .13 \\
\hline d. $1981-1986$ & $\begin{array}{l}-3.03^{-05} \\
\left\{2.66^{-05}\right\}\end{array}$ & $\begin{array}{l}.86^{* *} \\
(.01)\end{array}$ & & & & & $\begin{array}{l}.42^{* *} \\
(.06)\end{array}$ & $.90^{* *}$ \\
\hline e. $1977-1986$ & $\begin{array}{l}-7.22^{-05} \\
\left(4.45^{-05}\right\rangle\end{array}$ & $\begin{array}{l}.52^{* *} \\
(.02)\end{array}$ & & & & & $\begin{array}{l}1.24^{* *} \\
(.10)\end{array}$ & $.68^{* *}$ \\
\hline
\end{tabular}

$* \mathrm{p}<.05$

$* * \mathrm{p}<.01$

' Die Gleichungen a-c stellen OLS metrische Koeffizienten dar (Standardabweichungen). Die Gleichungen d und e sind gewichtete OLS metrische Koeffizienten (Standardabweichungen). 
Konkurrenz und Integration hin. Der dargelegte Befund widerspricht der anderen obengenannten Annahme, derzufolge die Ausländerbeschäftigung deutschen Arbeitnehmern zusätzliche Karrieremöglichkeiten verschafft. Der Effekt ist aber sehr klein. Wie sich schon für den Arbeitsmarkt insgesamt zeigte, reduziert die Anwesenheit einer durchschnittlichen Anzahl ausländischer Arbeitskräfte den Quotienten nur um ungefähr .09 bis .16 oder um vier bis sieben Prozent der Durchschnittswertes.

Die Tabelle 4 enthält die Ergebnisse einer ähnlichen Analyse, die sich aber auf die Bruttostundenlöhne männlicher Arbeiter in der untersten Leistungsgruppe stützt. Aus den genannten Gründen haben wir es nur mit der Industrie zu tun, so da $\beta$ die Zahl unserer Untersuchungseinheiten nur 222 beträgt. Dafür können hier zwei zusätzliche intervenierende Variablen kontrolliert werden, nämlich der Produktivitäszuwachs der Wirtschaftszweige und die durchschnittliche Firmengröße. Die Werte für diese Variablen repräsentieren nationale Durchschnittswerte. Alle Ergebnisse der Tabelle 4 zeigen, daß die Lohnhöhe in der Leistungsgruppe 3 durch Ausländerbeschäftigung nicht verringert wird. Im Gegenteil ist ein Anstieg zu beobachten. Er ist freilich sehr gering. Der größte Effekt zeigt sich im Jahr 1986. In einer Branche mit einer durchschnittlichen Zahl von ausländischen Ar- beitskräften, nämlich 1.926 und durchschnittlichen Bruttostundenlöhnen in der Leistungsgruppe 3, nämlich $\mathrm{DM} 14,51$ beträgt der Lohnanstieg nur DM 0,21 oder ein Prozent. Ein zehnprozentiger Zuwachs der Ausländerbeschäftigung geht einher mit einem Lohnzuwachs von nur drei Pfennig.

In die gleiche Richtung deuten die Ergebnisse über die Veränderungen im Zeitablauf. Berechnet man die Veränderungseffekte für die beiden letzten Gleichungen in Tabelle 4, dann erhält man:

D. 1981-1986 Lohn $=20.0+2.30^{-05}$ (ausländische männliche Arbeiter)

E. 1977-1986 Lohn $=19.2+1.89^{-04}$ (ausländische männliche Arbeiter)

Diese Befunde deuten kaum auf Diskriminierung hin. Auf sie wäre unter Umständen zu schließen, wenn die Ausländerbeschäftigung die durchschnittlichen Stundenverdienste sinken ließe. Im Widerspruch steht der genannte Befund aber auch zu der Annahme, zwischen deutschen und ausländischen Arbeitskräften bestünden Konkurrenzverhältnisse. Denn der neoklassischen Volkswirtschaftslehre und dem Marx'schen Theorem der industriellen Reservearmee zufolge müßte unter Konkurrenzverhältnissen ein zunehmendes Angebot ausländischer Arbeitskräfte die Löhne sinken lassen. Unser Befund widerspricht also sowohl der Annahme, es existierten Konkurrenzverhältnisse

Tabelle 4 Ausländerbeschäftigung (Zahl der ausländischen Nichtgelernten) und Bruttostundenverdienste in Leistungsgruppe 3 in den industriellen Länderwirtschaftszweigen $(N=222)^{1}$

Kontrollvariablen

Ausl. Lag Term Größe Wachstum Sektor Branche $\underset{\text { firmen- }}{\text { größe }}$ -
Arbeiter

\begin{tabular}{|c|c|c|c|c|c|c|c|c|c|}
\hline a. 1977 & $\begin{array}{r}6.76^{-05 *} \\
\left(2.75^{-05}\right)\end{array}$ & & $\begin{array}{l}-2.29^{-06} \\
\left(3.26^{-06}\right)\end{array}$ & $\begin{array}{l}-9.14^{-08} \\
\left(1.12^{-06}\right)\end{array}$ & $\begin{array}{l}-.70^{* *} \\
(.24)\end{array}$ & $\begin{array}{l}-.02 \\
(.01)\end{array}$ & $\begin{array}{c}.004 \\
(.002)\end{array}$ & $\begin{array}{l}11.23^{* *} \\
(1.11)\end{array}$ & $.42^{* *}$ \\
\hline b. 1981 & $\begin{array}{c}1.07^{-04 * *} \\
\left(3.64^{-05}\right)\end{array}$ & & $\begin{array}{l}-5.28^{-06} \\
\left(3.83^{-06}\right)\end{array}$ & $\begin{array}{c}-1.20^{-06} \\
\left(6.17^{-06}\right)\end{array}$ & $\begin{array}{l}-.86^{* *} \\
(.31)\end{array}$ & $\begin{array}{l}-.08^{* *} \\
(.02)\end{array}$ & $\begin{array}{l}.01 * * \\
(.004)\end{array}$ & $\begin{array}{l}19.45^{* *} \\
(2.27)\end{array}$ & $.42^{* *}$ \\
\hline c. 1986 & $\begin{array}{c}1.08^{-04} \\
\left(6.08^{-05}\right)\end{array}$ & & $\begin{array}{c}-1.56^{-06} \\
\left(5.16^{-06}\right)\end{array}$ & $\begin{array}{c}-1.13^{-05} \\
\left(1.04^{-05}\right)\end{array}$ & $\begin{array}{c}-1.50^{* *} \\
(.42)\end{array}$ & $\begin{array}{l}-.01 \\
(.01)\end{array}$ & $\begin{array}{l}.02^{* *} \\
(.005)\end{array}$ & $\begin{array}{l}14.26^{* *} \\
(1.03)\end{array}$ & $.44^{* *}$ \\
\hline d. $1981-1986$ & $\begin{array}{r}5.28^{-05 *} \\
\left(2.26^{-05}\right)\end{array}$ & $\begin{array}{l}.77^{* *} \\
(.06)\end{array}$ & & & & & & $\begin{array}{l}4.9^{* *} \\
(.72)\end{array}$ & $.67^{* *}$ \\
\hline e. $1977-1986$ & $\begin{array}{c}9.65^{-05 * *} \\
\left(2.66^{-05}\right)\end{array}$ & $\begin{array}{l}.49^{* *} \\
(.05)^{2}\end{array}$ & & & & & & $\begin{array}{l}9.8^{* *} \\
(.60)\end{array}$ & $.50^{* *}$ \\
\hline
\end{tabular}

$* p<.01$

** $\mathrm{p}<.001$

1 Die Gleichungen a-c stellen OLS metrische Koeffizienten dar (Standardabweichungen). Die Gleichungen d und $e$ sind gewichtete OLS metrische Koeffizienten (Standardabweichungen). 
Tabelle 5 Die Konzentration männlicher ausländischer Arbeiter in den Wirtschaftszweigen

\begin{tabular}{|c|c|c|c|c|c|c|}
\hline & \multicolumn{2}{|c|}{1977} & \multicolumn{2}{|c|}{1981} & \multicolumn{2}{|c|}{1986} \\
\hline & $\begin{array}{l}\text { Prozent aller } \\
\text { ausländischen } \\
\text { Arbeiter }\end{array}$ & $\begin{array}{c}\text { Prozent aller } \\
\text { deutschen } \\
\text { Arbeiter }\end{array}$ & $\begin{array}{c}\text { Prozent aller } \\
\text { ausländischen } \\
\text { Arbeiter }\end{array}$ & $\begin{array}{c}\text { Prozent aller } \\
\text { deutschen } \\
\text { Arbeiter }\end{array}$ & $\begin{array}{c}\text { Prozent aller } \\
\text { ausländischen } \\
\text { Arbeiter }\end{array}$ & $\begin{array}{c}\text { Prozent aller } \\
\text { deutschen } \\
\text { Arbeiter }\end{array}$ \\
\hline Industrie & 63 & 47 & 60 & 46 & 58 & 45 \\
\hline $\begin{array}{l}\text { Extraktive Bran- } \\
\text { chen mit bela- } \\
\text { stender Arbeit' }\end{array}$ & 21 & 15 & 21 & 15 & 18 & 13 \\
\hline $\begin{array}{l}\text { Ausländerkonzen- } \\
\text { tration in allen } \\
\text { Wirtschafts- } \\
\text { zweigen }{ }^{2}\end{array}$ & \multicolumn{2}{|c|}{8,8} & \multicolumn{2}{|c|}{8,7} & \multicolumn{2}{|c|}{8,5} \\
\hline
\end{tabular}

1 Hier sind die folgenden 11 Wirtschaftsbereiche eingeschlossen: Land- und Forstwirschaft, Fischerei, die verschiedenen Untergliederungen des Bergbaus, Eisen-und Stahlindustrie. Fleischverarbeitung, Bauindustrie, Reinigungsgewerbe.

$2\left(\sqrt{ } E\left({ }^{x 1} / x\right)^{2}\right) 100$.

wie derjenige ethnischer Diskriminierung. Er zwingt zu dem Schluß, daß Konkurrenz zwischen deutschen und ausländischen Arbeitskräften durch andere Strukturen als diejenigen ethnischer Diskriminierung unterbunden wird. Dafür gibt es tatsächlich auch zahlreiche Anhaltspunkte; sie werden weiter unten erörtert.

Die letzte Hypothese geht davon aus, daß Diskriminierung sich auch in der Verteilung der ausländischen Arbeitskräfte über die verschiedenen Wirtschaftszweige manifestieren müßte. Ausländer müßten in Wirtschaftszweigen mit ungünstigen Arbeitsplätzen konzentriert sein. Tabelle 5 zeigt, daß ausländische Arbeitskräfte in der Tat auch anders über die verschiedenen Wirtschaftszweige verteilt sind als deutsche. Sie finden Beschäftigung öfter in der Industrie und den extraktiven Zweigen der Wirtschaft. Die Arbeitsbedingungen sind hier weniger günstig, so kann man unterstellen. Falls auf dem Arbeitsmarkt Normen ethnischer Diskriminierung gelten, dann müßte die Konzentration der ausländischen Arbeitnehmer in diesen ungünstigeren Wirtschaftsbereichen im Verlauf unserer Untersuchungsperiode zunehmen, und zwar um so mehr, je länger die ethnozentrische Norm oder Attitüde Zeit hat, sich auszuwirken. Tatsächlich nimmt der Konzentrationsgrad jedoch $\mathrm{ab}$.

Wir benutzen einen Standardindex der Arbeitskräftekonzentration, der Konzentration als eine direkte Funktion der relativen Ungleichheit der Verteilung über die Einheiten (Länderwirtschaftszweige) wiedergibt, kontrolliert durch die rezipro- ke Funktion der Anzahl der Einheiten (Hirshman 1945), oder

$C=\left(\sqrt{ } \Sigma\left(X_{1} / X\right)\right)^{2} 100$,

dabei bedeutet $X_{1}$ die Gesamtzahl der ausländischen Arbeitskräfte im Wirtsschaftszweig i und X die Gesamtzahl der ausländischen Arbeitskräfte in allen Wirtschaftszweigen. Es zeigt sich: Die Verteilung der Ausländer über die verschiedenen Zweige der Wirtschaft gleicht sich derjenigen der deutschen Arbeitnehmer an. Ein Indikator für den Konzentrationsgrad auf dem gesamten Arbeitsmarkt kann das verdeutlichen. Unser Indikator hätte den Wert Null für Gleichverteilung deutscher und ausländischer Arbeitnehmer und den Wert 100 für die Konzentration aller ausländischen Arbeitnehmer in einem Wirtschaftszweig. Bildet man die tatsächlichen Verhältnisse auf diesem Kontinuum $a b$, dann ergibt sich ein Wert von nur 8,0, also einer, der sehr nahe an dem Pol der Gleichverteilung liegt. Er nimmt im Untersuchungszeitraum $\mathrm{ab}$.

\section{Diskussion}

Die dargelegten Befunde werfen ein Licht auf Effekte der Ausländerbeschäftigung und auf die Bedeutung ethnischer Diskriminierung auf dem Arbeitsmarkt. Das dabei erkennbare Bild läßt sich im Hinblick auf die Unterschichtungshypothese und im Hinblick auf ethnisch bedingte Karrieremobilität so skizzieren: 
Ausländerbeschäftigung führt unter männlichen Vollzeitbeschäftigten zu einer Ausweitung nichtgelernter Arbeit, also ungünstigerer Arbeitsverhältnisse. Das könnte auf Diskriminierung schließen lassen. Aber die Schicht der nichtgelernten Arbeit wird nicht nur durch ausländische, sondern auch durch deutsche männliche Vollzeitarbeiter erweitert. Die genannten Effekte sind aber so gering, $\mathrm{daß}$ sie eher als Hinweise auf Stabilität der überkommenen Verhältnisse zu deuten sind. Der These ethnisch bedingter Unterschichtung widerspricht auch, daß die Ausländerbeschäftigung die durchschnittlichen Bruttostundenlöhne, die in der Leistungsgruppe 3 an deutsche und ausländische Männer gezahlt werden, keinesfalls hat sinken lassen.

Gegen die Annahme ethnischer Kategorienbildung sprechen auch die Ergebnisse zur Karrieremobilität. Ausländischen Arbeitnehmern gelingt auch beruflicher Aufstieg. Zudem ist nicht zu beobachten, daß deutsche Arbeitnehmer infolge der Zuwanderung ausländischer Arbeitskräfte häufiger berufliche Karriere gemacht hätten.

Ausländische Beschäftigte scheiden in größerem Umfang aus dem Arbeitsmarkt aus und sind auch häufiger als Deutsche von Arbeitslosigkeit betroffen. Dabei spielt vermutlich auch Diskriminierung eine Rolle. Mit den hier verfügbaren Daten läßt sich das aber nicht überprüfen.

Schließlich, es gelingt den ausländischen Arbeitnehmern, aus den Wirtschaftszweigen mit besonders ungünstigen Arbeitsbedingungen herauszukommen. Ihre Verteilung über die Wirtschaftszweige gleicht sich derjenigen ihrer deutschen Kollegen an.

Die hier dargelegten Befunde zur Arbeitsmarktintegration stimmen mit Ergebnissen anderer Studien aus Deutschland, den USA und der Schweiz überein. So fínden Köhler/Preisendörfer in einer methodisch aufwendigen Einzelfallstudie, daß sich deutsche und ausländische Arbeiter „,beim Aufstieg in höhere Hierarchieebenen und erst recht in ihrem Lohnzuwachs kaum unterscheiden“ (1988: 272). Mit zunehmender Dauer der Betriebszugehörigkeit und Erfahrung gelingt es ihnen, in die Positionen von Springern, Einstellern und Gruppenführern aufzusteigen. Hinweise auf Aufstiegsmobilität unter Einwanderern finden sich auch bei König/Schultze/Wessel (1985).

Die vergleichsweise hohen Arbeitsplatzverluste unter ausländischen Beschäftigten erklären sich, empirischen Studien zufolge, unter anderem aus dem niedrigen Qualifikationsniveau, der Beschäf- tigung in Wirtschaftsbereichen mit besonders hohem Arbeitsplatzverlust infolge Konjunkturabhängigkeit und wirtschaftlichem Strukturwandel, aus mangelnden Sprach- und Qualifikationsvoraussetzungen für den Wechsel in den expandierenden tertiären Bereich (Dietz 1988). Aufhebungsverträge und Abfindungen werden von ausländischen Arbeitskräften bereitwilliger akzeptiert als von Deutschen. Dazu trägt der Wunsch bei, nach Hause zurückzukehren, oder aber die Furcht, als Ausländer diskriminiert zu werden und deswegen über kurz oder lang den Arbeitsplatz zu verlieren, oder beides (Köhler/Preisendörfer 1988).

In der amerikanischen Literatur finden sich Studien, die den Folgen der Einwanderung für die Löhne der Einheimischen nachgehen. Auch in den USA, so zeigt sich, wurden die Löhne der Alteingesessenen durch Zuwanderer kaum geschmälert (Borjas 1986; Grossman 1982; DeFreitas/Marshall 1984). Andere Studien weisen darauf hin, daß sich gravierendere Effekte erst bei hoher Konzentration von Zuwanderern in bestimmten Arbeitsmarktsektoren ergeben (Tyrchniewicz/Schuh 1969; Wise 1974; De Freitas/Marshall 1984). Zu diesem Schluß kommen auch Borjas/Tienda (1987) und Borjas (1986) in einer ausgedehnten Literaturauswertung.

Untersucht wurde auch die These, Einwanderung münze sich für die Eingesessenen in Aufstiegsvorteile um. Ausgangspunkt bildet dabei die Theorie des dualen Arbeitsmarktes und die Annahme, die Alteingesessenen würden in den günstigeren Sektor 1 befördert, wenn die Zuwanderer in den Sektor 2 des Arbeitsmarktes einrückten (Piore 1979). Aber die Beweise für diese Annahme sind schwach. Zum einen ist kritisiert worden, da $\beta$ die These vom dualen Arbeitsmarkt der Realität kaum gerecht wird (vgl. Dickens/Lang 1985). Nur wenn das Angebot an einheimischen Arbeitskräften extrem klein ist, könne mit Segmentationseffekten gerechnet werden (Borjas/Tienda 1987). Schließlich ist mit marktfremden Größen zu rechnen, die einem Einfluß der Immigration auf die Lage einheimischer Arbeitskräfte entgegenstehen. Wie die Volkswirtschaftslehre weiß und Unternehmer klagen, sind Löhne und andere Arbeitsbedingungen unelastisch. Sie hängen ab von organisatorischen, kulturellen oder gesetzlichen Regelungen, die nicht einfach durch eine Vermehrung des Arbeitskräfteangebots außer Kraft zu setzen sind (Greenwood/McDowell 1986). Niedrig ist schließlich auch die Angebotselastizität der Arbeitskräftenachfrage, was die Möglichkeit von Segmentationseffek- 
ten ebenfalls herabsetzt (Beck 1980; Hamermesh 1976; Hamermesh/Grant 1979; Greenwood 1983). So ergeben sich also auch aus einer breiten Diskussion in den USA deutliche Hinweise darauf, daß das institutionelle Gefüge des Arbeitsmarktes durch Einwanderung nicht wesentlich verändert wird.

Diesen Befunden entsprechen auch die Daten einer Studie, die Hoffmann-Nowotny (1973) in der Schweiz für den Zeitraum 1930-1960 gewonnen hat. Zwischen 1941 und 1960 hat sich die Zahl der ausländischen Arbeitskräfte in der Schweiz nahezu verfünffacht, der Anteil der Arbeiter ist aber bei 50 Prozent geblieben. Trotz Einwanderung hat die berufliche Schichtenpyramide in dem hier betrachteten Ausschnitt ihre Form also gehalten. Die Einwanderer, so zeigt sich weiter, nehmen die Arbeitsplätze vor allem in den unteren Bereichen des beruflichen Schichtensystems ein.

Hoffmann-Nowotny wertet die beobachtete Stabilität des beruflichen Schichtensystems als Ausdruck einer Unterschichtung, die durch Einwanderung bedingt sei. Ohne Einwanderung, so lautet das Argument, hätten die untersten Schichten der Berufspyramide schrumpfen müssen. Denn geschrumpft seien sie auch in den Jahren zuvor, bis 1941 eine Expansion der Einwanderung einsetzte. Das zusätzliche Angebot an Arbeitskräften hätte produktivitätsschwachen Unternehmen das Überleben ermöglicht, Rationalisierungsfortschritte verlangsamt und folglich die Beseitigung manueller Arbeit unterbunden. Überzeugend ist diese Begründung nicht. Ausländerbeschäftigung rettet nicht nur die Betriebe mit Grenzproduktivität, sondern vermehrt auch die Profite der übrigen und schafft also zusätzliche Mittel für technische Innovationen. Ob der technische Fortschritt aber überhaupt die unqualifizierte Arbeit verschwinden läßt, ist eine offene Frage. Entsprechende Erwartungen finden sich bereits in den technizistischen Utopien des 19. Jahrhunderts; sie konnten aber bis heute empirisch nicht belegt werden.

Kehren wir in die Bundesrepublik zurück. Ethnische Kategorien, so läßt sich aus den dargelegten Befunden schließen, sind in der Arbeitswelt kaum entstanden. Seit Beginn der Gastarbeiterpolitik gab es einen stabilen Konsensus zwischen Regierung, Arbeitgebern und Gewerkschaften über ein Nebeneinander von Regeln der Diskriminierung und solchen der Integration: Die Interessen deutscher Arbeitnehmer sollten dominieren, wenn es um die Frage der Öffnung und Schließung der Außengrenzen des Arbeitsmarktes geht, innerhalb des Arbeitsmarktes dagegen sollten Ausländer und Deutsche gleichgestellt sein (vgl. Dohse 1981; Bielefeld 1989).

Darauf hatten vor allem die Gewerkschaften gedrungen. Die Kombination von Inländerprimat nach außen und Gleichbehandlung im Innern entspricht ihren Funktionsbedingungen. Denn ihre Verhandlungsmacht hängt vom Vertretungsmonopol für alle Arbeitnehmer ab. Ethnische Konkurrenz würde ihre Position schwächen. Deswegen müssen sie darauf bestehen, daß ausländische Arbeitskräfte nicht außerhalb tarifvertraglicher $\mathrm{Re}$ gelungen beschäftigt werden und daß in den Tarifverträgen nicht nach Kriterien der Staatsangehörigkeit unterschieden wird. Von Ausnahmen abgesehen, konnten sich die Gewerkschaften mit ihrer Forderung auch durchsetzen. Anders als in Deutschland sind die Gewerkschaften in den USA zum Träger sozialer Abgrenzungen nach ethnischen Kriterien geworden. Sie sind fraktioniert und können nur die Interessen begrenzter Arbeitnehmergruppen vertreten. Für sie wurden ethnische Ressentiments zu einer Organisationsressource (vgl. Bonacich 1979). Der Inländerprimat hatte es den deutschen Gewerkschaften erleichtert, bei ihren Mitgliedern die Zustimmung für die Öffnung des Arbeitsmarktes zu gewinnen. Denn er versprach, daß ausländische Arbeitskräfte den deutschen das Risiko der Arbeitslosigkeit abnehmen würden. Zugleich sollte er die Gewerkschaften vor dem Druck der ,industriellen Reservearmee schützen.

Auch die Bundesregierung verband mit der Gastarbeiterpolitik Erwartungen, denen eine ethnische Kategorienbildung innerhalb des Arbeitsmarktes widersprochen hätte. Die Anwerbung ausländischer Arbeitskräfte sollte nicht zuletzt dem schnellen Anstieg der Löhne entgegenwirken und damit verbunden der Inflation. Die angestrebte lohndämpfende Konkurrenz und die Verschonung der Einheimischen vor dem Risiko der Arbeitslosigkeit setzen aber voraus, daß inländische und ausländische Arbeitskräfte gegeneinander austauschbar und insofern also gleich sind. Darüber hinaus war die Bundesregierung mit den Regierungen jener Länder konfrontiert, aus denen die Gastarbeiter gekommen waren und die die Interessen ihrer Bürger vertraten.

Für die Unternehmer schließlich hätten rechtliche Diskriminierungen zwischen deutschen und ausländischen Arbeitern staatlichen Dirigismus bedeutet, der die Personalpolitik nur belastet. Sie haben sich deswegen staatlichen Versuchen entge- 
gengestellt, bestehende Arbeitsverträge mit ausländischen Arbeitskräften zugunsten deutscher Arbeitsloser zu lösen (Dohse 1981: 504f).

So stimmten die Sachwalter des Staates, der Wirtschaft und der Arbeitnehmer in dem Grundsatz überein, daß die Ausländer zu den gleichen formalen Bedingungen wie Deutsche beschäftigt werden müßten. Tatsächlich sind die ausländischen Arbeitskräfte rechtlich auch weitgehend gleichgestellt. Die Bestimmungen des Tarifvertragsrechts, des Urlaubsrechts, des Arbeitsschutz- und Arbeitszeitrechts, des Mutterschutzes und des arbeitsgerichtlichen Rechtsschutzes gelten für Ausländer in gleicher Weise wie für Deutsche. Auch im Streikrecht, im Kündigungsschutzgesetz, in der Bezahlung von Überstunden, der Zahlung außertariflicher betrieblicher Leistungen, Prämien und Beihilfen werden keine Unterschiede gemacht. Zur Betriebsratswahl sind sie aktiv und passiv legitimiert. Der organisierte Arbeitsmarkt, so läßt sich daraus folgern, ist formal jedenfalls durch die Masseneinwanderung ausländischer Arbeitskräfte nur wenig modifiziert worden (Sieveking 1990; Pitzen-Meynert/Maier/Schlicker/Bermig 1988).

Es gibt freilich auch Diskriminierung. Das ist dort offenkundig, wo gesetzliche Regelungen Ausländern den Zugang zu bestimmten Berufspositionen verschließen. Kraft Gesetzes wird die Ausländereigenschaft hier $z u$ einer entscheidenden Größe der Statusallokation. So sind im Bereich des öffentlichen Dienstes Ausländer wegen der Bestimmung des Artikels 33 GG (Berufsbeamtentum) diskriminiert. Es gibt weiterhin berufsregelnde Spezialgesetze mit besonderen Vorschriften, nach denen die Erteilung der notwendigen Erlaubnis grundsätzlich auf Deutsche beschränkt ist. Deren Zahl scheint aber abzunehmen, und so gehört auch das kuriose Beispiel der Hackfleischverordnung, die die Herstellung von Hackfleisch deutschen Staatsbürgern vorbehielt, der Vergangenheit an. Eine andere gesetzliche Diskriminierung besteht darin, daß die Aufenthaltserlaubnis mit dem Sperrvermerk versehen werden kann, der die Aufnahme einer selbständigen Erwerbstätigkeit verwehrt. An diese Entscheidung der Ausländerbehörden sind die $\mathrm{Ge}$ werbebehörden gebunden, die gewerberechtliche Genehmigungen zuteilen. Die betreffenden Ausländer sind dann von selbständiger oder vergleich barer unselbständiger Erwerbstätigkeit ausgeschlossen. Die Erwerbsstatistik zeigt, daß Ausländer seltener als deutsche Staatsbürger selbständig sind, daß sich ihr Anteil an den Selbständigen aber mehr als verdreifacht hat. Er betrug 1970 1,6 Pro- zent und 1988 5,1 Prozent (Wirtschaft und Statistik 2, 1990: 90; vgl. auch Morokvasic/Phizacklea/Rudolph 1986).

\section{Zusammenfassung}

Gefragt wurde nach ethnisch bedingter Unterschichtung der Berufspyramide und nach ethnischer Diskriminierung in der beruflichen Statusallokation. Untersucht wurde der Bereich männlicher vollzeitbeschäftigter Arbeiter. Da dieser Sektor des Arbeitsmarktes besonders dicht reguliert ist und von den Interessenverbänden besonders aufmerksam kontrolliert wird, müßten sich Normen ethnischer Diskriminierung hier am ehesten zeigen.

Die Studie stützt sich auf Daten aus der Statistik der sozialversicherungspflichtig beschäftigten Arbeitnehmer und auf solche aus der Industriestatistik. Zusätzliche Evidenz erbrachte eine Übersicht über institutionelle Regelungen, die den Status von Inländern und Ausländern im Arbeitsleben bestimmen, sowie eine Literaturanalyse.

Ausländerbeschäftigung geht einher mit einer geringfügigen Verschiebung der Relation von Facharbeit zu nichtgelernter Arbeit. Das deutet auf Unterschichtung hin, der Effekt ist jedoch sehr gering. Von dieser Verschiebung sind nicht nur Ausländer, sondern auch deutsche Arbeitskräfte betroffen. Gegen die Unterschichtungshypothese spricht schließlich auch, daß Ausländerbeschäftigung die Bruttostundenverdienste in der Leistungsgruppe 3 nicht hat sinken lassen.

Es ergeben sich keine Anhaltspunkte dafür, daß die Ausländerbeschäftigung Deutschen zusätzliche Karrieremöglichkeiten verschafft hat. $\mathrm{Zu}$ beobachten ist, daß einer Anzahl von Einwanderern der berufliche Aufstieg in Meisterpositionen gelungen ist.

Schließlich zeigte sich, daß die Verteilung der Ausländer über die verschiedenen Wirtschaftszweige sich derjenigen der deutschen Arbeitskräfte angeglichen hat.

Der aufenthaltsrechtliche und arbeitserlaubnisrechtliche Status der großen Mehrzahl der Einwanderer, auch derjenigen von außerhalb der EG, hat sich entscheidend verfestigt. Sie können ihre Existenz in der BRD insofern als gesichert betrachten. Differenzen im Rechtsstatus deutscher und ausländischer Arbeitnehmer gibt es, ihre Wirkungen scheinen aber begrenzt. Der Tendenz zu formaler rechtlicher Gleichstellung scheint im großen und 
ganzen auch eine zu materialer Gleichbehandlung zu entsprechen.

Ein Caveat sei zum Schluß wiederholt: Wichtige Formen möglicher Diskriminierung können in dieser Studie nicht sichtbar werden, sei es, weil die hier verwandten Indikatoren zu grob sind, sei es, weil sie nur einen Sektor des Arbeitsmarktes repräsentieren.

\section{Literaturverzeichnis}

Baker, D./Lenhardt, G., 1988: Ausländerintegration, Schule und Staat. Kölner Zeitschrift für Soziologie und Sozialpsychologie 1: 40-61.

Baker, D./Lenhardt, G., 1990: Inclusion or Exclusion? Labor Market Consequences of Immigration to the Federal Republic of Germany. Research Report. Washington, D.C.: The Catholic University of America; Berlin: Max-Planck-Institut für Bildungsforschung.

Beck, E. M., 1980: Labor Unionism and Racial Income Inequality. A Time-Series Analysis of the Post-World War II in Period. American Journal of Sociology 4: 791-814.

Bendix, R., 1982: Die Staatsbürgerschaft der unteren Klassen. S. 103-119 in: R. Bendix, Freiheit und historisches Schicksal. Heidelberger Max Weber Vorlesungen 1981. Frankfurt/M.: Suhrkamp.

Bielefeld, U., 1989: Zum Strukturwandel des ,Ausländerproblems" in der BRD. Gewerkschaftliche Monatshefte 40: 393-404.

Bonacich, E., 1979: A Theory of Ethnic Antagonism: The Split Labor Market Theory. American Sociological Review 37: 547-559.

Borjas, G. J., 1986: The Sensitivity of Labor Demand Functions to Choice of Dependent Variables. Review Econ. Statist. 61: 58-66.

Borjas, G. J./Tienda, M., 1987: The Economic Consequences of Immigration. Science 235: 645-651.

DeFreitas, G./Marshall, A., 1984: Immigration and Wage Growth in U.S. Manufacturing in the 1970s. S. 148-156 in: B. D. Dennis (Hrsg.), Proceedings of the Thirty-Sixth Annual Meeting, Dec. 28-30, 1983. IRRA.

Dickens, W./Lang, K., 1985: A Test of Dual Labor Market Theory. American Economic Review 75: 792-805.

Dietz, F., 1988: Strukturwandel auf dem Arbeitsmarkt. Mitteilungen aus der Arbeitsmarkt- und Berufsforschung 1: 115-136.

Dohse, K., 1981: Ausländische Arbeiter und bürgerlicher Staat. Königstein/Ts.: Hain.

Dombois, R., 1980: Arbeitsplatz Volkswagenwerk. Manuskript. Bremen.

Elias, N., 1989: Studien über die Deutschen. Frankfurt/ M.: Suhrkamp.

Esser, H./Friedrichs, J. (Hrsg.), 1990: Generation und Identität. Opladen: Westdeutscher Verlag.
Gillmeister, H./Kurthen, H./Fijalkowski, J., 1989: Ausländerbeschäftigung in der Krise. S. 197-215 in: $M$. Bolle/B. Strümpel (Hrsg.), Beiträge zur Sozialökonomik der Arbeit. Band 21. Berlin: Edition Sigma.

Greenwood, M. J., 1983: The Economics of Mass Migration from Poor to Rich Countries: Leading Issues of Fact and Theory. American Economic Review 73: 173-177.

Greenwood, M. J./McDowell, J. M., 1986: The Factor Market Consequences of Immigration. Journal of Economic Literature 24: 1738-1772.

Grossman, J. B., 1982: The Substitutability of Natives and Immigrants in Productions. Review Econ. Statist. 64: 596-603.

Hamermesh, D. S., 1976: Econometric Studies of Labor Demand and Their Application to Policy Analysis. J. Human Res. 11: 507-525.

Hamermesh, D. S./Grant, J., 1979: Econometric Studies of Labor - Labor Substitution and Their Implications for Policy. J. Human Res. 14: 518-542.

Hanushek, E. A./Jackson, J. E., 1977: Statistical Methods for Social Scientists. New York: Academic Press.

Heckmann, F., 1981: Die Bundesrepublik: Ein Einwanderungsland. München: Deutsches Jugendinstitut.

Hoffmann-Nowotny, H. J., 1973: Soziologie des Fremdarbeiterproblems. Stuttgart: Enke.

Kmenta, J., 1977: Elements of Econometrics. New York: Macmillan.

Köhler, C./Preisendorfer, P., 1988: Innerbetriebliche Arbeitsmarktsegmentation in Form von Stamm- und Randbelegschaft. Mitteilungen aus der Arbeitsmarktund Berufsforschung 2: 268-277.

König, P./Schultze, G./Wessel, R., 1985: Zur Situation der ausländischen Arbeitnehmer und ihrer Familienangehörigen in der BRD. Repräsentativuntersuchung 1980. Bonn: Forschungsinstitut der Friedrich-EbertStiftung. Arbeitsgruppe Ausländerforschung und Ausländerpolitik.

Kühne, P./Schäfer, H., 1986: Soziale Ungleichheit von Ausländern. S. $118-137$ in: H. W. Franz et al. (Hrsg.), Neue alte Ungleichheiten. Opladen: Westdeutscher Verlag.

Lenhardt, G., 1990: ,Ethnische Identität" und gesellschaftliche Rationalisierung. Prokla $20 \mathrm{Nr}$. 79: 132-154.

Lüttinger, P., 1986: Der Mythos der schnellen Integration. Eine empirische Untersuchung zur Integration der Vertriebenen und Flüchtlinge in der BRD bis 1971. Zeitschrift für Soziologie 15: 20-36.

Marshall, T. H., 1965: Class, Citizenship and Social Development. Garden City, N. Y.

Meier-Braun, K.-H., 1988: Integration und Rückkehr? Mainz: Matthias-Grünewald-Verlag.

Morokvasic, M./Phizacklea, A./Rudolph, H., 1986: Small Firms and Minority Groups: Contradictory Trends in the French, German, and British Clothing Industry. International Sociology 3: 1-21.

Oberndörfer, D., 1990: Wert der Staatsbürgerschaft in Geschichte und Gegenwart. S. 55-65 in: Senatsver- 
waltung für Gesundheit und Soziales - Ausländerbeauftragte (Hrsg.), Doppelte Staatsbürgerschaft - ein europäischer Normalfall. Dokumentation des Internationalen Fachkongresses, 8.-20. Oktober 1989. Berlin: Senatsverwaltung für Gesundheit und Soziales Die Ausländerbeauftragte (Selbstverlag).

Piore, M. J., 1979: Birds of Passage: Migrant Labor and Industrial Societies. New York: Cambridge University Press.

Pitzen-Meynert, B./Maier, H.-W./Schlicker, M./Bermig, K., 1988: Gutachten über ausländerdiskriminierende Vorschriften im deutschen Recht. Manuskript. Bonn.

Portes, A./Bach, R. L., 1980: Immigrant Earnings: Cuban and Mexican Immigrants in the United States. International Migration Review 14: 315-341.

Reimann, H./Reimann, R. (Hrsg.), 1987²: Gastarbeiter. Analysen und Perspektiven eines sozialen Problems. Opladen: Westdeutscher Verlag.

Sassen, S., 1988: The Mobility of Labor and Capital. Cambridge, Mass.: Cambridge Univ. Pr.

Senatsverwaltung für Gesundheit und Soziales - Ausländerbeauftragte (Hrsg.), 1990: Doppelte Staatsbürgerschaft - ein europäischer Normalfall. Dokumentation des Internationalen Fachkongresses, 18. -20. Oktober 1989. Berlin: Senatsverwaltung für Gesundheit und Soziales - Die Ausländerbeauftragte (Selbstverlag).

Sieveking, K., 1990: Arbeitsmigranten als Nicht-Bürger S. 11-33 in: Senatsverwaltung für Gesundheit und Soziales - Ausländerbeauftragte (Hrsg.), Doppelte Staatsbürgerschaft - ein europäischer Normalfall. Dokumentation des Internationalen Fachkongresses, 18. -20. Oktober 1989. Berlin: Senatsverwaltung für Gesundheit und Soziales - Die Ausländerbeauftragte (Selbstverlag).
Snowden, L. L., 1990: Collective Versus Mass Behavior: A Conceptual Framework for Temporary and Permanent Migration in Western Europe and the United States. International Migration Review 24: 577-590.

Szydlik, M., 1990: Die Segmentierung des Arbeitsmarkts in der BRD. (Beiträge zur Sozialökonomie der Arbeit, hrsg. von M. Bolte, und B. Strümpel, 24). Berlin: Edition Sigma.

Statistisches Bundesamt, 1977-1986a: Sozialversicherungspflichtig beschäftigte Arbeitnehmer. Fachserie 1, Reihe 4.2. 1977 bis 1986. Wiesbaden. (Die Daten liegen auch auf Datenträgern vor; auf die wurde zurückgegriffen.)

Statistisches Bundesamt, 1977-1986b: Arbeiterverdienste in der Industrie. Fachserie 16, Reihe 2.1. 1977 bis 1986. Wiesbaden.

Treibel, A., 1988: Engagement und Distanzierung in der westdeutschen Ausländerforschung. Stuttgart: Enke.

Trommer, L., 1991: Demographische Entwicklung und Bildungsbeteiligung von Ausländern. Unveröffentlichtes Manuskript.

Trychniewicz, E. W./Schuh, E. G., 1969: Econometric Analysis of the Agricultural Labor Market. Amer. J. Agr. Econ. 51: 770-787.

Wallerstein, I., 1974: The Modern World-System. New York: Academic Press.

Wirtschaft und Statistik, hg. vom Statistischen Bundesamt. Wiesbaden (laufend).

Wise, D. E., 1974: The Effect of the Bracero on Agricultural Production in California. Econ. Inquiry 12: 547-558.

Zapf, W./Brachtl, A., 1984: Gastarbeiter und deutsche Bevölkerung. S. 286-306 in: W. Glatzer/W. Zapf (Hrsg.), Lebensqualität in der BRD. Frankfurt/M.; New York. 